\title{
Bandwidth and Power Allocation for Cooperative Strategies in Gaussian Relay Networks
}

\author{
Ivana Maric Member, IEEE and Roy D. Yates Member, IEEE
}

\begin{abstract}
In a relay network with a single sourcedestination pair, we examine the achievable rates with amplifyand-forward (AF) relaying strategy. Motivated by applications in sensor networks, we consider power-constrained networks with large bandwidth resources and a large number of nodes. We show that the AF strategy does not necessarily benefit from the large available bandwidth. We characterize the optimum AF bandwidth and show that transmitting in the optimum bandwidth allows the network to operate in the linear regime where the achieved rate increases linearly with the available network power. We then present the optimum power allocation among the AF relays. The solution, which can be viewed as a form of maximum ratio combining, indicates the favorable relay positions in the network.

Motivated by the large bandwidth resources we further consider a network that uses orthogonal transmissions at the nodes. While the above result for the optimum bandwidth still holds, a different set of relays should optimally be employed. In this case, the relay power solution can be viewed as a form of water-filling.

The optimum AF bandwidth and the relay powers can be contrasted to the decode-and-forward (DF) solution. In a network with unconstrained bandwidth, the DF strategy will operate in the wideband regime to minimize the energy cost per information bit [1], [2]. The wideband DF strategy requires again a different choice of relays; in the case of orthogonal signaling, the data should be sent through only one DF relay [3] . Thus, in general, in a large scale network, a choice of a coding strategy goes beyond determining a coding scheme at a node; it also determines the operating bandwidth as well as the best distribution of the relay power.
\end{abstract}

\section{INTRODUCTION}

Although the capacity of the single-relay channel [4] and consequently of wireless relay networks remains unknown, several coding strategies have been proposed [5]-[9]. Most of these strategies require the relay to decode reliably the source message before forwarding and we refer to them as decode-and-forward (DF) [10].

In another strategy that does not require reliable decoding at the relays, called amplify-and-forward (AF) [10], a relay forwards the scaled version of the received noisy copy of the source signal. Hence, the data is sent through only two-hops with no cooperation among relays. Under the assumption of uncoded transmission at the source, it was shown that the two-hop AF strategy achieves the asymptotic capacity in a relay network in the limit as the number of relays becomes large [11], [12]. It was further shown that in a random network, power efficiency of such a strategy increases with the number of relays [13].
In this paper, we revisit the AF strategy in a network with many relays and a single source-destination pair. Motivated by the sensor networks, we consider networks in which the power is a limiting resource. Compared to the power, the bandwidth in such a network is plentiful. Operating in the wideband regime then seems like a right choice: at the expense of using a large number of degrees of freedom, the transmit energy per bit can be reduced. However, the minimum energy cost per information bit in a general relay network is unknown [14]. We show that the AF strategy does not necessarilly benefit from the available large bandwidth. We characterize the optimum AF bandwidth and show that transmitting in the optimum bandwidth allows the network to operate in a linear regime where the achieved rate increases linearly with transmit power.

We then present the optimum power allocation among the AF relays. The solution can be viewed as a form of maximum ratio combining (MRC) with the powers being proportional to the equivalent channel gains that depend not only on the relay-destination channel gains, but also on the source-relay links.

The requirement of coherent combining of the signals transmitted from the different nodes may be too demanding in practice. Furthermore, a network with a large bandwidth available, can support orthogonal signaling at the nodes that precludes coherent combining at a receiver. To evaluate the performance of a two-hop network that does not benefit from coherent combining, we also consider a relay network model with orthogonal transmissions at the nodes. We show that the above result for the optimum bandwidth applies to this channel model as well. Furthermore, for the case of AF orthogonal transmissions, we again identify the best subset of AF relay nodes. The optimum relay power solution in this case can be viewed as a form of water-filling.

The optimum AF bandwidth and the relay powers can be contrasted to the DF solution. In a network with unconstrained bandwidth, the DF strategy will operate in the wideband regime to minimize the energy cost per information bit [1], [2]. The wideband DF strategy requires again a different choice of relay nodes; in the case of orthogonal signaling, the data should be sent through one DF relay that is in the "best" position in the network [3]. Thus, in general, a choice of a coding strategy goes beyond determining a coding scheme at a node; it also determines the operating bandwidth as well as the best distribution of the relay power. 


\section{System Model}

We consider a wireless Gaussian network with a single source, labeled node 0 , the destination, labeled node $M+1$, and $M$ relays that dedicate their resources to relaying information for the source. We consider two bandwidth allocations in the given network:

1) Shared bandwidth. All the relays transmit in a common bandwidth $W^{(1)}$.

2) Orthogonal channels. Every node is assigned an orthogonal channel of bandwidth $W^{(2)}$.

We adopt a discrete-time Gaussian channel model [15] and let the vector $\mathbf{X}[n]=\left[X_{0}[n], \ldots, X_{M}[n]\right]^{T}$ denote the channel inputs in time slot $n$. The input $X_{0}[n]$ depends on the source message and the input $X_{m}[n]$ at relay $m$ depends on its past outputs $X_{m}[n]=f_{m}\left(Y_{m}[1], \ldots, Y_{m}[n-1]\right)$.

In such a network, we consider two-hop forwarding strategies in which, relays use only the information received from the source to choose their channel inputs and forward the messages to the destination. In the first hop, the source transmits. The channel output at relay $m$ is

$$
Y_{R m}[n]=\sqrt{\alpha_{m}} X_{0}[n]+Z_{R m}[n]
$$

and at the destination,

$$
Y[n]=\sqrt{\beta_{0}} X_{0}[n]+Z[n],
$$

where $\sqrt{\alpha_{m}}$ and $\sqrt{\beta_{0}}$ are the source-relay $m$ and sourcedestination channel gain, respectively and $Z[n]$ is a zeromean Gaussian random variable with variance $N_{0} / 2$. In the second hop, relays transmit. In shared bandwidth, the channel output at the destination is

$$
Y[n]=\sum_{m=1}^{M} \sqrt{\beta_{m}} X_{m}[n]+Z[n]
$$

However, when relays use orthogonal channels,

$$
\mathbf{Y}[n]=\mathbf{B X}[n]+\mathbf{Z}[n]
$$

where $\mathbf{B}=\operatorname{diag}\left(\begin{array}{lll}\sqrt{\beta_{0}} & \cdots & \sqrt{\beta_{M}}\end{array}\right)$ and $X_{0}[n]=0 . \mathbf{Z}$ is a Gaussian noise vector with covariance matrix $\mathbf{K}=\sigma^{2} \mathbf{I}_{M+1}$.

Using the cut-set Theorem [15, Thm. 14.10.1], it was shown [11] that the capacity of this network is upper bounded by $\log M$, given that there is a dead zone around the source that contains no relays.

As in [11], we consider the amplify-and-forward protocol at the relays, in which the noisy version of the source input $X_{0}$ received at relay $m, 1 \leq m \leq M$ is amplified and forwarded with a unit delay. For amplification gain $b_{m} \geq 0$, in time slot $n$, relay $m$ transmits

$$
X_{m}[n]=\sqrt{b_{m}}\left(\sqrt{\alpha_{m}} X_{0}[n-1]+Z_{R m}[n-1]\right) .
$$

In this paper, rather than considering the power constraint imposed on each transmitter, we assume that the total power budget of $p$ [Watts] is allocated to the network. The constraint is on the total power rather than on the power per dimension because DF and AF will not in general operate in the same bandwidth. Further, such a constraint allows a power allocation among the nodes such that $E\left[\mathbf{X}^{T} \mathbf{X}\right] \leq$ $p / 2 W^{(i)}, i=1,2$.

\section{OPTIMUM BANDWIDTH ALLOCATION}

We next consider the rates achievable with the AF strategy. Let $p_{m}$ denote the power at node $m$ and let $\mathbf{p}=\left[\begin{array}{lll}p_{0} & \ldots & p_{M}\end{array}\right]^{T}$ be the power allocation at all nodes. Vector $\mathbf{P}=\left[\begin{array}{lll}P_{0} & \ldots & P_{M}\end{array}\right]^{T}$ denotes nodes' powers per dimension and $P_{m}=p_{m} / 2 W^{(i)} i=1,2$. The amplification gain $b_{m}$ is chosen such that the transmit power at node $m$ is $p_{m}$ and is found from (5) to be

$$
b_{m}=\frac{P_{m}}{\alpha_{m} P_{0}+N_{0} / 2} \quad m=1, \ldots M
$$

The achievable rates, given by the maximum mutual information between the channel input and the output can be found from the result [16] to be

$$
I_{A F}^{(i)}(\mathbf{P})=\frac{1}{2} \log \left[1+\frac{P_{0}}{N_{0} / 2}\left(\beta_{0}+G^{(i)}(\mathbf{P})\right)\right]
$$

for $i=1,2$ where

- For the shared bandwidth,

$$
G^{(1)}(\mathbf{P})=\frac{\left(\sum_{m=1}^{M} \sqrt{\frac{\alpha_{m} \beta_{m} P_{m}}{\alpha_{m} P_{0}+N_{0} / 2}}\right)^{2}}{\left(\sum_{m=1}^{M} \frac{\beta_{m} P_{m}}{\alpha_{m} P_{0}+N_{0} / 2}+1\right)} .
$$

- For orthogonal channels,

$$
G^{(2)}(\mathbf{P})=\sum_{m=1}^{M} \frac{\alpha_{m} \beta_{m} P_{m}}{\alpha_{m} P_{0}+\beta_{m} P_{m}+N_{0} / 2} .
$$

Rates (7) are normalized by the number of dimensions utilized by a node rather than by the total number of dimensions in the channel. For $G^{(i)}(\mathbf{P})=0$, (7) becomes the rate achieved in the single-user channel, by a direct source transmission at power $P_{0}$. Thus, we can view $G^{(i)}(\mathbf{P})$ as the gain obtained by employing the AF relays. The difference in the AF gains (8) and (9) comes from the coherent combining of the relay signals transmitted in a shared bandwidth, which is forfeited in the orthogonal channel system. The analysis presented in this section, however, applies to both cases and we therefore drop the $(i)$ superscript. We next consider the total rate achieved by the AF strategy

$$
r_{A F}=2 W I_{A F}(\mathbf{P}) \quad \text { bits } / \mathrm{s},
$$

where $I_{A F}$ is given by (7). As $W$ becomes large, we observe that $G(\mathbf{p} / W)$ decreases to zero and therefore

$$
\lim _{W \rightarrow \infty} r_{A F}=\lim _{W \rightarrow \infty} W \log \left(1+\frac{\beta_{0} p_{0}}{N_{0} W}\right)=\frac{\beta_{0} p_{0}}{N_{0} \ln 2} \quad \text { bits } / \mathrm{s}
$$

which is the rate achieved in the wideband regime by the source transmission. Therefore, there is no benefit from AF relays transmitting in the wideband regime. This behavior was previously observed in [17] in a parallel Gaussian 
network with two relays. Except for the somewhat trivial case in which the source is in a favorable position compared to all the relays, the rate $r_{A F}$ generally decreases for large $W$.

To characterize the optimum AF bandwidth, we formulate the AF power/bandwidth relay problem as

$$
\begin{aligned}
& r^{*}=\max _{\mathbf{P}, W} 2 W I_{A F}(\mathbf{P}) \\
& \text { subject to } 2 W \sum_{m=0}^{M} P_{m} \leq p, \\
& \quad \mathbf{P} \geq 0, \\
& \quad 0 \leq W \leq W_{\max } .
\end{aligned}
$$

We assume that $W_{\max }$ is sufficiently large to allow the network to operate in the wideband regime. Let $\left(\mathbf{P}^{*}, W^{*}\right)$ denote the optimum power and bandwidth allocation that achieves $r^{*}$ in (12). We first observe that, to achieve nonzero rate in (12), it has to hold that $W^{*}>0$ and $P_{0}^{*}>0$. Furthermore, constraint (12a) is always binding. Depending on the values of the channel gains, a solution to problem (12) may be a direct source transmission, that is, $P_{m}^{*}=0$ for $m=1, \ldots, M, W^{*}=W_{\max }$ and $P_{0}^{*}$ given by (12a). Otherwise, there will be a set of $0<K \leq M$ relays employing the AF strategy. Given $\mathbf{P}^{*}$, it will be convenient to relabel the nodes such that $m \in\{1, \ldots, K\}$ relays are the active transmitters with powers $P_{m}^{*}>0$ while $P_{m}^{*}=0$, for $m \in\{K+1, \ldots, M\}$. The Lagrangian in (12) is

$$
\Lambda=2 W I_{A F}(\mathbf{P})-\mu\left(2 W \sum_{m=0}^{M} P_{m}-p\right)
$$

The fact that rate $r_{A F}$ is decreasing with $W$ for large $W$, implies that $W^{*}<W_{\max }$. Since, in addition $W^{*}>0$, the solution to (12) is never on the boundary (12c). By the KuhnTucker conditions, this implies

$$
\frac{\partial \Lambda}{\partial W}=2 I_{A F}\left(\mathbf{P}^{*}\right)-2 \mu \sum_{m=0}^{K} P_{m}^{*}=0
$$

From (14), we obtain the Lagrange multiplier

$$
\mu=\frac{I_{A F}\left(\mathbf{P}^{*}\right)}{\sum_{m=0}^{K} P_{m}^{*}} \text {. }
$$

For nodes $k=0, \ldots, K$ with non-zero transmitter powers, the Kuhn-Tucker conditions are

$$
\frac{\partial \Lambda}{\partial P_{k}}=2 W^{*}\left[\frac{\partial I_{A F}\left(\mathbf{P}^{*}\right)}{\partial P_{k}}-\mu\right]=0, \quad k=0, \ldots, K .
$$

From (15) and (16),

$$
\frac{\partial I_{A F}\left(\mathbf{P}^{*}\right)}{\partial P_{k}}=\mu=\frac{I_{A F}\left(\mathbf{P}^{*}\right)}{\sum_{m=0}^{K} P_{m}^{*}}, \quad k=0, \ldots, K .
$$

The optimum power allocation $\left(P_{0}^{*}, \ldots, P_{K}^{*}\right)$ can then be determined from $K+1$ equations given by (17), and is independent of $r$ and $W^{*}$. We present the solution for the optimum relay powers in the next section. The optimum bandwidth can be determined such that the solution lies on the feasibility region boundary $(12 \mathrm{a})$ :

$$
W^{*}=\frac{p}{2 \sum_{m=0}^{K} P_{m}^{*}} .
$$

From (12), (15) and (18),

$$
r^{*}=2 W^{*} I_{A F}\left(\mathbf{P}^{*}\right)=\frac{I_{A F}\left(\mathbf{P}^{*}\right)}{\sum_{m=0}^{K} P_{m}^{*}} p=\mu p .
$$

We thus proved the following:

Theorem 1: The AF relay problem (12) has an optimum solution in which the optimum bandwidth $W^{*}$, the maximum rate $r^{*}$ and the total power $p$ have a linear relationship.

\section{OPTIMUM RELAY POWER ALLOCATION}

We next consider a subproblem of (12) that determines the optimum relay powers per dimension, for any given source power $P_{0}$. We consider the shared bandwidth case first.

\section{A. Shared Bandwidth}

Given a source power $P_{0}$, we let

$$
\gamma_{m}=\frac{\beta_{m}}{\alpha_{m} P_{0}+N_{0} / 2} .
$$

To maximize the rate (7) over the relay powers $\hat{\mathbf{P}}=$ $\left[\begin{array}{lll}P_{1} & \ldots & P_{M}\end{array}\right]^{T}$, we maximize the $\mathrm{AF}$ gain (8)

$$
\begin{gathered}
\max _{\hat{\mathbf{P}}} \frac{\left(\sum_{m=1}^{M} \sqrt{\alpha_{m} \gamma_{m} P_{m}}\right)^{2}}{\sum_{m=1}^{M} \gamma_{m} P_{m}+1} \\
\text { subject to } \sum_{m=1}^{M} P_{m} \leq P_{R}, \\
\hat{\mathbf{P}} \geq 0
\end{gathered}
$$

where $P_{R}=p / 2 W-P_{0}$ is the power allocated to the relays. To solve (21), we first argue that the solution is always on the boundary (21a). To see that, consider a feasible solution $\overline{\mathbf{P}}$ such that $\sum_{m=1}^{M} \bar{P}_{m}<P_{R}$. Then, there exist a constant $K>1$ and a feasible solution $\mathbf{P}^{\prime}=K \overline{\mathbf{P}}$ such that $\mathbf{P}^{\prime}$ is on the boundary $K \sum_{m=1}^{M} \bar{P}_{m}=P_{R}$. Furthemore, it is easy to verify that $G\left(\mathbf{P}^{\prime}\right)>G(\overline{\mathbf{P}})$. We can thus let the constraint (21a) be satisfied with equality. The objective function (21) becomes

$$
G(\hat{\mathbf{P}})=\frac{\left(\sum_{m=1}^{M} \sqrt{\alpha_{m} \gamma_{m} P_{m}}\right)^{2}}{\sum_{m=1}^{M}\left(\gamma_{m}+1 / P_{R}\right) P_{m}}
$$

A solution to problem (21) can be found by representing the objective function (22) in the form of Rayleigh quotient that would then be maximized [18]. A simpler approach, however, can be used by introducing variables $\mathbf{z}=\left[z_{m}\right]$

$$
z_{m}=\sqrt{\gamma_{m}+1 / P_{R}} \sqrt{P_{m}}, \quad m=1, \ldots, M
$$


and a vector of coefficients $\mathbf{d}=\left[d_{m}\right]$

$$
d_{m}=\sqrt{\frac{\alpha_{m} \gamma_{m}}{\gamma_{m}+1 / P_{R}}}, \quad m=1, \ldots, M
$$

Problem (21) can then be represented in a vector form

$$
\max _{\mathbf{z}} \frac{\left(\mathbf{d}^{T} \mathbf{z}\right)^{2}}{\mathbf{z}^{T} \mathbf{z}} .
$$

Applying the Schwartz inequality, the solution to (25) is $\mathbf{z}^{*}=k \mathbf{d}$ where the constant $k$ can be found from (21a) and (23). We get the optimum powers in the MRC form as

$$
P_{m}^{*}=\frac{P_{R} \delta_{m}}{\sum_{k=1}^{M} \delta_{k}} .
$$

where we defined

$$
\delta_{m}=\frac{\alpha_{m} \gamma_{m}}{\left(1+\gamma_{m} P_{R}\right)^{2}}
$$

The AF gain (8) becomes

$$
G^{(1)}\left(P_{0}, P_{R}\right)=\sum_{m=1}^{M} \frac{\alpha_{m} \beta_{m} P_{R}}{\alpha_{m} P_{0}+\beta_{m} P_{R}+N_{0} / 2} .
$$

Next Lemma follows by comparing AF gains (28) and (9).

Lemma 1: For any given powers $\left(P_{0}, P_{R}\right)$ at the source and at the AF relays, signaling in shared bandwidth outperforms orthogonal signaling.

Given the relay powers (26), the AF power/bandwidth problem (12) for any given $W$ reduces to

$$
\begin{gathered}
\max _{P_{0}, P_{R}} I_{A F}\left(P_{0}, P_{R}\right) \\
\text { subject to } P_{0}+P_{R} \leq P_{T}=\frac{p}{2 W}, \\
P_{0}, P_{R} \geq 0 .
\end{gathered}
$$

Lemma 2: There exists a unique optimum solution $\left(P_{0}^{*}(W), P_{R}^{*}(W)\right)$ to $(29)$.

The proof for the Lemma follows from the concavity of the objective function on the boundary.

Given $\left(P_{0}^{*}(W), P_{R}^{*}(W)\right)$, the AF power/bandwidth problem (12) reduces to maximizing the rate with respect to bandwidth for $0 \leq W \leq W_{\max }$

$$
r^{*}(W)=\max _{W} 2 W I_{A F}(W) .
$$

\section{B. Numerical Results}

The relay powers (26) are shown in Figures 1-4 for a scenario of $M=2500$ relays positioned on a $100 \times 100$ square grid. The source and the destination are positioned on the two opposite sides of the grid. The propagation exponent $n=2$ was chosen.

For large source power $P_{0}$, relay powers are shown in Figure 1. In this case, the received SNR at the relays is high and the network MAC side from the relays to the destination limits the performance. The relays that have a better channel to the destination are employed. Figure 2 shows the opposite case of a small power $P_{0}$ and a high power $P_{R}$. We observe a reversed relay power allocation compared to the previous case, as the network tries to improve the broadcast side performance by choosing the relays with high received SNR. Figure 3 shows the powers for larger values of $P_{0}$ and $P_{R}$. Finally, Figure 4 shows the relay powers when the network operates in a low SNR-regime due to small $P_{0}$ and $P_{R}$.

We next look at the solution to the AF relay problem (12). For a given network power budget $p$ [Watts], the best powers $\left(P_{0}^{*}, P_{R}^{*}\right)$ and the bandwidth $W^{*}$ are found numerically. As the power budget in the network is varied, the achieved rate and the optimum bandwidth, shown in Figure 5, vary linearly, as promised by Theorem1. The powers $\left(P_{0}^{*}, P_{R}^{*}\right)$ stay constant for all values of $p$, a behavior we observed in (17). Due to the symmetry of the network in the experiment, the values of powers $P_{0}^{*}$ and $P_{R}^{*}$ are very close to each other.

\section{Orthogonal Channels}

We next identify the best subset of AF relays and their powers for the case of orthogonal signaling. Given a source power $P_{0}$, we let

$$
\gamma_{m}=\sqrt{\frac{\alpha_{m} \beta_{m}}{\alpha_{m} P_{0}+N_{0} / 2}} .
$$

Again, to maximize the rate (7) over the relay powers $\hat{\mathbf{P}}$, we maximize the $\mathrm{AF}$ gain (9)

$$
\begin{array}{r}
\max _{\hat{\mathbf{P}}} \sum_{m=1}^{M} \frac{\alpha_{m} \gamma_{m}^{2} P_{m}}{\alpha_{m}+\gamma_{m}^{2} P_{m}} \\
\text { subject to } \sum_{m=1}^{M} P_{m} \leq P_{R}, \\
\hat{\mathbf{P}} \geq 0 .
\end{array}
$$

From the Kuhn-Tucker conditions, the solution to (32) is in the form of water-filling

$$
P_{m}^{*}=\frac{\alpha_{m}}{\gamma_{m}}\left[\frac{1}{\sqrt{\eta}}-\frac{1}{\gamma_{m}}\right]^{+} \quad m=1, \ldots, M
$$

where $\eta$ is the Lagrange multiplier and is found such that constraint (32a) is satisfied with equality. Once again, the best choice of relays varies with the transmit source power. We conclude that the AF relay network, depending on whether it operates in shared or orthogonal channels, will require two different relay power allocations as given by (26) and (33). Employing a wideband DF strategy with orthogonal signaling results in yet another solution that requires a use of a single relay [3]. Thus, in general, a choice of a coding strategy goes beyond determining a coding scheme at a node; it also determines the operating bandwidth as well as the best distribution of the relay power.

\section{REFERENCES}

[1] S. Verdú, "Spectral efficiency in the wideband regime," IEEE Trans. on Information Theory, vol. 48, no. 6, pp. 1319-1343, June 2002. 


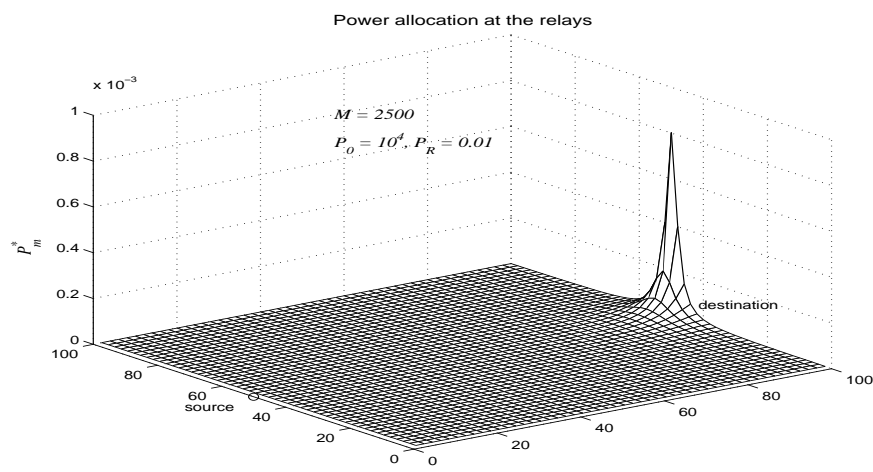

Fig. 1. Relay powers for $P_{0}=10^{4}, P_{R}=0.01$. Due to a small power available to the relays, the MAC side limits the network performance. The solution chooses relays that have a better relay-destination channel.

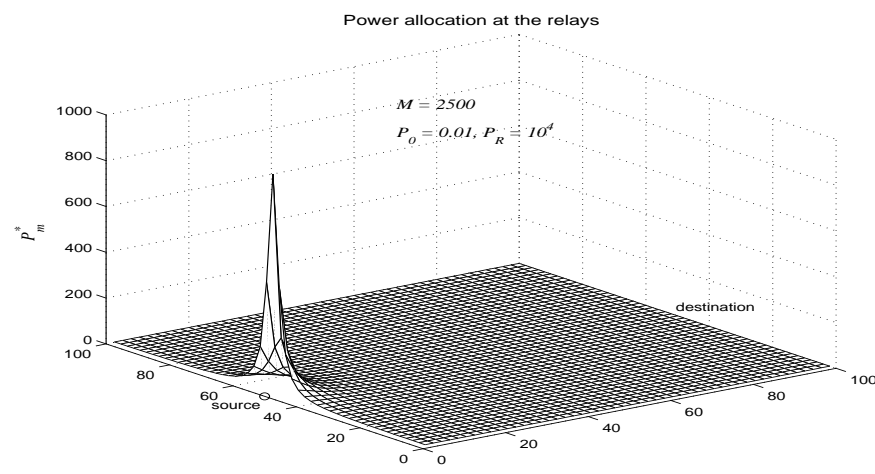

Fig. 2. Relay powers for $P_{0}=0.01, P_{R}=10^{4}$. Relays that are closer to the source are employed.

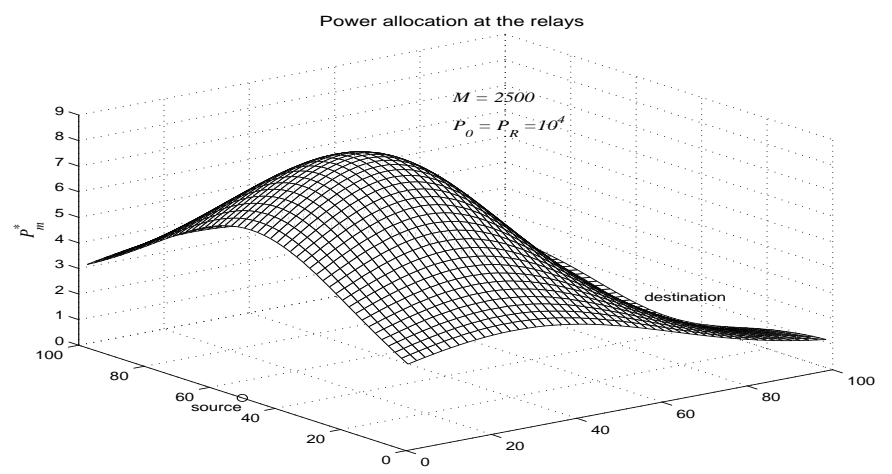

Fig. 3. Relay powers for $P_{0}=P_{R}=10^{4}$ and $N_{0} / 2=1$.

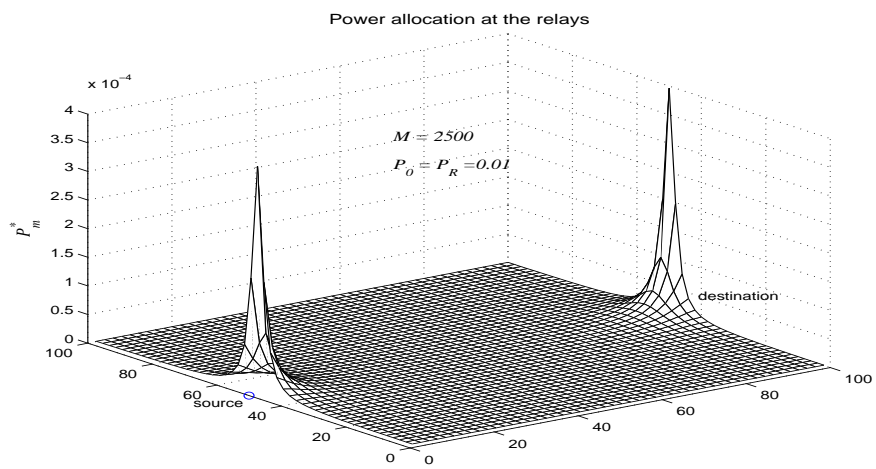

Fig. 4. Relay powers for $P_{0}=P_{R}=0.01$ and $N_{0} / 2=1$.
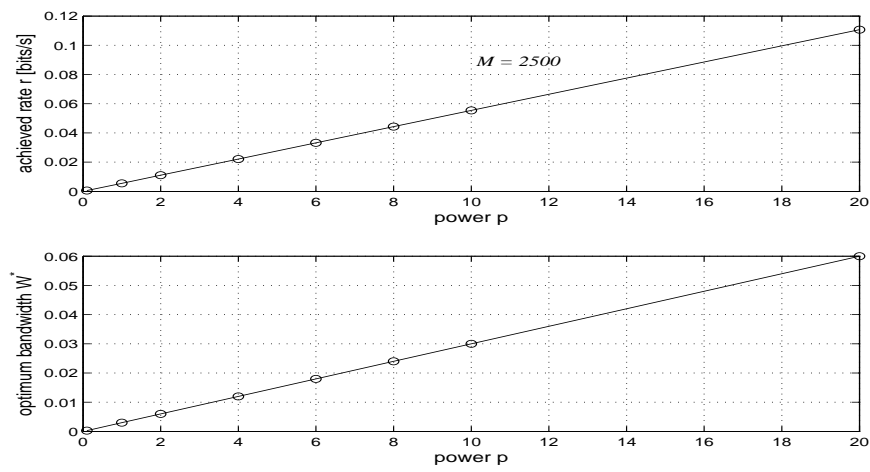

Fig. 5. Achieved rate and the optimum bandwidth as a function of the network power budget $p$ [Watts].

[2] O. Oyman and A. J. Paulraj, "Spectral efficency of relay networks in the power-limited regime," in Proc. of Allerton Conference on Communication, Control and Computing, Sept. 2004.

[3] I. Maric and R. D. Yates, "Forwarding strategies for gaussian parallelrelay networks," in Proc. of IEEE International Symposium on Information Theory, June 2004.

[4] E. C. van der Meulen, "Three-terminal communication channels," $A d v$. Appl. Prob., vol. 3, pp. 120-154, 1971.

[5] T. Cover and A. E. Gamal, "Capacity theorems for the relay channel," IEEE Trans. on Information Theory, vol. 25, no. 5, pp. 572-584, Sept. 1979.

[6] F. M. J. Willems, "Information theoretical results for the discrete memoryless multiple access channel," in Ph.D. Thesis, Katholieke Universiteit Leuven, Leuven, Beligum, Oct. 1992.

[7] L.-L. Xie and P. R. Kumar, "A network information theory for wireless communication: Scaling laws and optimal operation," IEEE Trans. on Information Theory, submitted.

[8] G. Kramer, M. Gastpar, and P. Gupta, "Cooperative strategies and capacity theorems for relay networks," IEEE Trans. Inform. Theory, submitted, Feb. 2004.

[9] —- "Capacity theorems for wireless relay channels," in Proc. of the Allerton Conference on Communications, Control and Computing, Monticello, IL, Oct. 2003.

[10] J. N. Laneman, D. N. C. Tse, and G. W. Wornell, "Cooperative diversity in wireless networks: efficient protocols and outage behavior," IEEE Trans. on Information Theory, submitted.

[11] M. Gastpar and M. Vetterli, "On the capacity of wireless networks: The relay case," in Proc. of INFOCOM'02, June 2002.

[12] _ , "On asymptotic capacity of gaussian relay networks," in Proc. of International Symposium on Information Theory (ISIT'02), June 2002.

[13] A. Dana and B. Hassibi, "On the power efficiency of sensory and ad-hoc wireless networks," IEEE Trans. Inform. Theory, submitted.

[14] G. Caire, D. Tuninetti, and S. Verdú, "Suboptimality of TDMA in the low-power regime," IEEE Trans. on Information Theory, submitted, Sept. 2002.

[15] T. Cover and J. Thomas, Elements of Information Theory. John Wiley \& Sons, Inc., 1991.

[16] E. Telatar, "Capacity of multi-antenna gaussian channels," in Europ. Trans. Telecommunications, Nov. 1997.

[17] B. E. Schein, "Distributed coordination in network information theory," in Ph.D thesis, Massachusetts Institute of Technology, Sept. 2001.

[18] G. Strang, Linear Algebra and its applications. Harcourt Brace Jovanovich College Publishers, 1988. 